\title{
Advanced Laser Retroreflectors for Astrophysics and Space Science
}

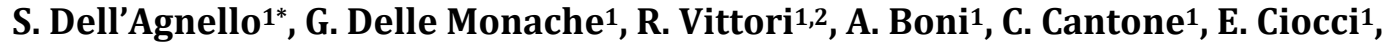

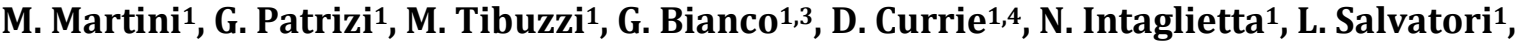 \\ C. Lops ${ }^{1}$, S. Contessa1 ${ }^{1}$, L. Porcelli' ${ }^{1}$, C. Mondaini' ${ }^{1}$, P. Tuscano' ${ }^{1}$, M. Maiello ${ }^{1}$ \\ ${ }^{1}$ Istituto Nazionale di Fisica Nucleare-Laboratori Nazionali di Frascati (INFN-LNF), Frascati, Italy \\ ${ }^{2}$ Aeronautica Militare Italiana, Italy \\ ${ }^{3}$ Agenzia Spaziale Italiana-Centro di Geodesia Spaziale (ASI-CGS), Matera, Italy \\ ${ }^{4}$ University of Maryland at College Park, MD, USA \\ Email: ${ }^{*}$ Simone.DellAgnello@Inf.infn.it
}

Received 18 December 2014

\section{Abstract}

We developed advances laser retroreflectors for solar system exploration, geodesy and for precision test of General Relativity (GR) and new gravitational physics: a micro-reflector array (INRRI, Instrument for landing-Roving laser Retroreflectors Investigations), a midsize reflector array for the European Earth Observation (EO) program, Copernicus (CORA, COpernicus laser Retroreflector Array), a large, single-retroreflector (MoonLIGHT, Moon Laser Instrumentation for General relativity High accuracy Tests). These laser retroreflectors will be fully characterized at the SCF_Lab (Satellite/lunar/GNSS laser ranging/altimetry Cube/microsat Characterization Facilities Laboratory), a unique and dedicated infrastructure of INFN-LNF (www.Inf.infn.it/esperimenti/etrusco/). Our research program foresees several activities: 1) Developing and characterizing the mentioned laser retroreflector devices to determine landing accuracy, rover positioning during exploration and planetary/Moon's surface georeferencing. These devices will be passive, laser wavelengthindependent, long-lived reference point. INRRI will enable the performance of full-column measurement of trace species in the Mars atmosphere by future space-borne lidars. These measurements will be complementary to highly localized measurements made by gas sampling techniques on the Rover or by laser back-scattering lidar techniques on future orbiters and/or from the surface. INRRI will also support laser and quantum communications, carried out among future Mars Orbiters and Mars Rovers. This will be possible also because the INRRI laser retroreflectors will be metal back-coated and, therefore, will not change the photon polarization. The added value of INRRI is its low mass, compact size, zero maintenance and its usefulness for any future laser altimetry, ranging, communications, atmospheric lidar capable Mars orbiter, for virtually decades after the end of the Mars surface mission, like the Apollo and Lunokhod lunar laser retroreflectors. MoonLIGHT and INRRI are proposed for landings on the Moon (two Google Lunar X Prize Missions, namely Moon Express; Russia's Luna-27 mission, as well as others under consideration/negotiation, also with the help of ASI, ESA and other partnerships); 2) Precision tests of GR with LLR to MoonLIGHT reflectors. Development of new fundamental gravity physics models and study of experimental constraints to these models use also laser ranging and laser reflectors throughout the

*Corresponding author.

How to cite this paper: Dell'Agnello, S., Delle Monache, G., Vittori, R., Boni, A., Cantone, C., Ciocci, E., Martini, M., Patrizi, G., Tibuzzi, M., Bianco, G., Currie, D., Intaglietta, N., Salvatori, L., Lops, C., Contessa, S., Porcelli, L., Mondaini, C., Tuscano, P. and Maiello, M. (2015) Advanced Laser Retroreflectors for Astrophysics and Space Science. Journal of Applied Mathematics and Physics, 3, 218-227. http://dx.doi.org/10.4236/jamp.2015.32032 
solar system: extension of general relativity to include Spacetime Torsion, Non-Minimal Coupling between matter and curvature (so-called " $f_{1}(R)+f_{2}(R)$ " theories, or NMC gravity); 3) Extension of program to: Mars, Phobos and Deimos, Jupiter and Saturn icy/rocky moons, Near Earth Asteroids.

\section{Keywords}

\section{General Relativity, Satellite Laser Ranging (SLR), Lunar Laser Ranging (LLR), Cube Corner Retroreflectors (CCR)}

\section{Introduction}

The SCF_Lab (Satellite/lunar/GNSS laser ranging and altimetry and CubeSat/microsat Characterization Facility Laboratory) of INFN-LNF is designed to cover virtually LRAs (Laser Retroreflector Arrays) of CCRs (Cube Corner Retroreflectors) for missions in the whole solar system, with a modular organization of its instrumentation, two redundant SCF (SCF_Lab Characterization Facilities), and an evolutionary measurement approach, including customization and potentially upgrade on-demand (see http://www.lnf.infn.it/esperimenti/etrusco/ for a general description).

The SCF_Lab is dedicated to the characterization and modeling of the space segment of SLR (Satellite Laser Ranging [1]-[4]), LLR (Lunar Laser Ranging, [5]-[12]) and PLRA (Planetary Laser Ranging and Altimetry) for industrial and scientific applications. A description of the different applications in SLR, LLR, and PLRA can be found at http://www.Inf.infn.it/conference/laser2012/. The SCF_Lab consists of two OGSE called SCF (property of INFN) and SCF-G (which doubles our metrology capabilities for applications to Global Navigation Satellite System, GNSS, property of INFN and of ASI). Views of the SCF are shown in Figure 1 and Figure 2. The SCF is very versatile for its large number of measurement ports (side and back), very long horizontal translations and capabilities for LLR and PLRA CCR payloads. The SCF-G is optimized for GNSS.

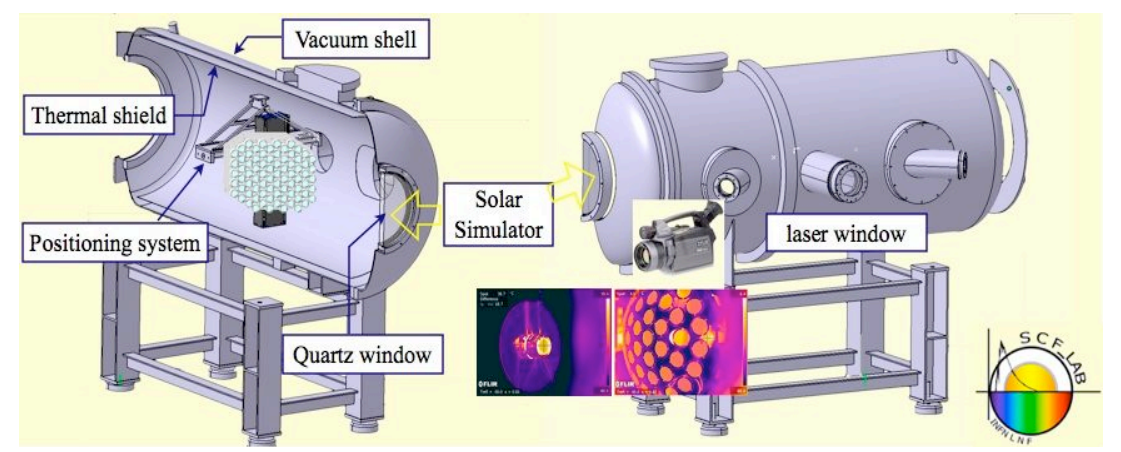

Figure 1. SCF cryostat, IR thermograms of CCRs under test, IR camera SCF_Lab logo.
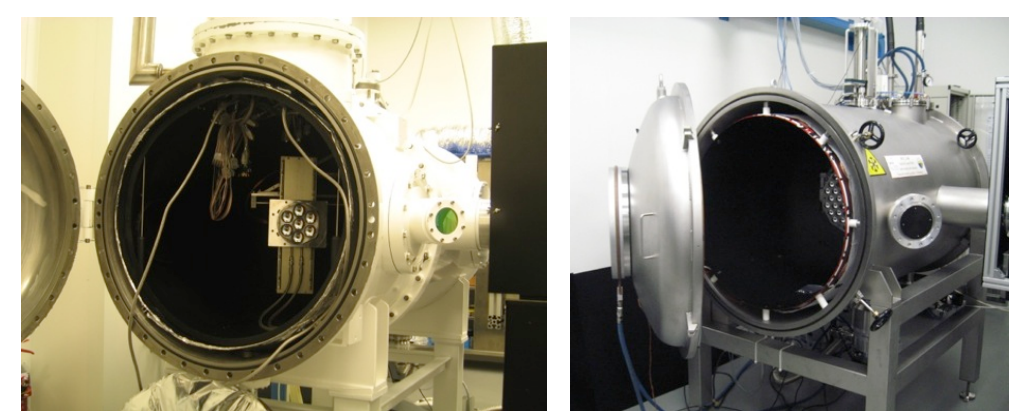

Figure 2. Left: SCF cryostat with GRA-H (GNSS Retroreflector Array with Hollow CCRs); IR port at right with a Ge window. Right: SCF-G cryostat with the GRA of solid CCRs; IR port at right with black cover on. 
Together with the SCF and SCF-G, we developed new industry-standard thermal-optical-vacuum tests to characterize and model the detailed optical performance and thermal behavior in representative space conditions (SCF-Test, background intellectual property of INFN). The latter is described in detail elsewhere [1] [2]. Here we only recall its key features:

- Laboratory-simulated space conditions. Concurrent/integrated:

- Dark/cold/vacuum

- Two Sun/Albedo AM0 simulators and Earth IR simulator

- Non-invasive IR and contact thermometry

- Laser interrogation and sun perturbation at varying angles

- Payload thermal control, roto-translations

- GCO, GNSS Critical Orbit (worst-case thermal and optical behavior).

- Deliverables/Retroreflector Key Performance Indicators (KPIs) measured in the above lab-simulated space conditions:

- Thermal behavior ( $\tau_{\mathrm{CCR}}$, thermal relaxation time) with probes and IR thermometry

- Optical response 1: Far Field Diffraction Pattern (FFDP)

- Optical response 2: (near-field) Wave front Fizeau Interferogram (WFI).

- Integrated thermal-optical simulations both of the test data and of orbit configurations data.

The SCF/SCF-GAM0 sun simulators have up to $45 \mathrm{~cm}$ diameter. The simulators can be used together on the SCF (from the front solar window and from the back port), where they allow for the performance of the full space environmental test of CubeSats of MicroSats capable of modeling both the thermal inputs of the Sun (Figure 3) and of the albedo (spectrum of Figure 3 properly scaled down).

We also have a vibration- and air-turbulence insensitive WFI measuring instrument to characterize the WFI of CCR during SCF-Tests (in addition to separate, independent and redundant FFDP measurements of the CCRs). This can be done with different laser polarizations (linear and circular).

We developed next-generation laser retroreflectors for: a flat, large LRA for satellite navigation, GRA (GNSS Retroreflector Array); a micro-reflector array for solar system exploration and geodesy, INRRI (Instrument for landing-Roving laser Retroreflectors Investigations); a midsize reflector array for Earth Observation (EO) and exploration, CORA (COpernicus laser Retroreflector Array); a single, large retroreflector fro LLR, MoonLIGHT (Moon Laser Instrumentation for General relativity High accuracy Tests) for the precision test of General Relativity (GR) [3] [5]-[12] and new gravitational physics [13]-[16]. These are being designed, built and characterized at the SCF_Lab.

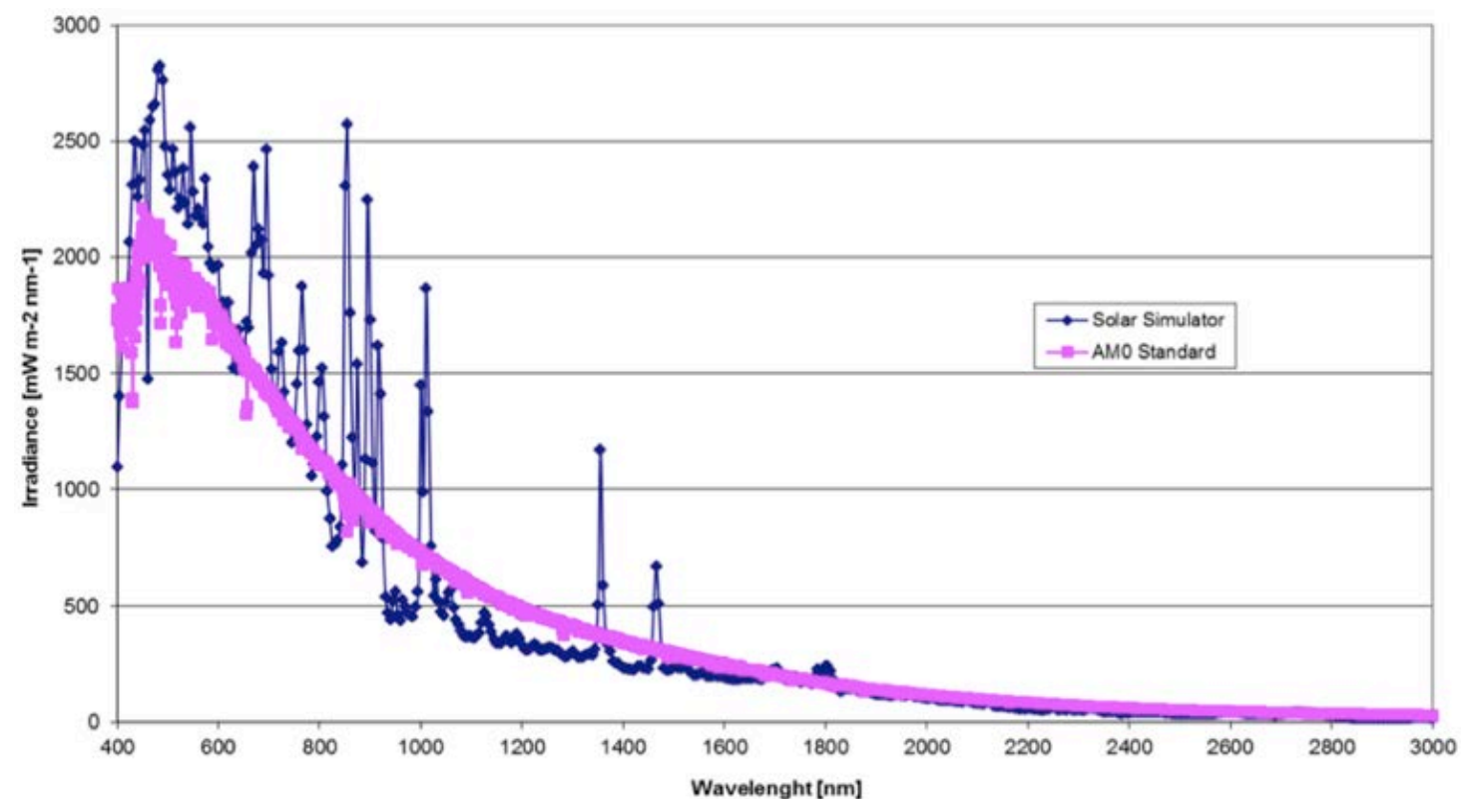

Figure 3. Spectrum of the SCF solar simulator (45 cm diameter) compared to the AM0 standard sun in space. 
We use LAGEOS (LAser GEO dynamics Satellite) and Apollo LRAs as reference payloads standards of the ILRS (International Laser Ranging Service; see http://ilrs.gsfc.nasa.gov). The SCF-Test of an engineering model of a polar sector of LAGEOS on loan at the SCF_Lab by NASA is described in [1] and [2]. The thermal SCF-Test of a flat-shape $3 \times 3$ CCR matrix with LAGEOS/Apollo CCRs and mounting scheme, built by the SCF_Lab, is described in [3] and [4].

\section{LLR and Gravitational Physics}

For the Moon we propose both Moon LIGHT (shown in Figure 4) and INRRI. The latter will be described in detail in the section "SOLAR SYSTEM LRA". In the following we describe Moon LIGHT. Precision GR tests and search for new gravitational physics are carried out with Apollo, Lunokhod LRAs and with MoonLIGHT. Current GR test [5]-[12] with LLR include:

- Parametrized Post-Newtonian parameter

- Weak Equivalence Principle

- Strong Equivalence Principle

- Time Variation of the Gravitational Constant

- Inverse Square Law-Yukawa potential

- Geodetic Precession.

Table 1 shows current limits on precision tests of GR from [9] and improvements achievable with MoonLIGHT.
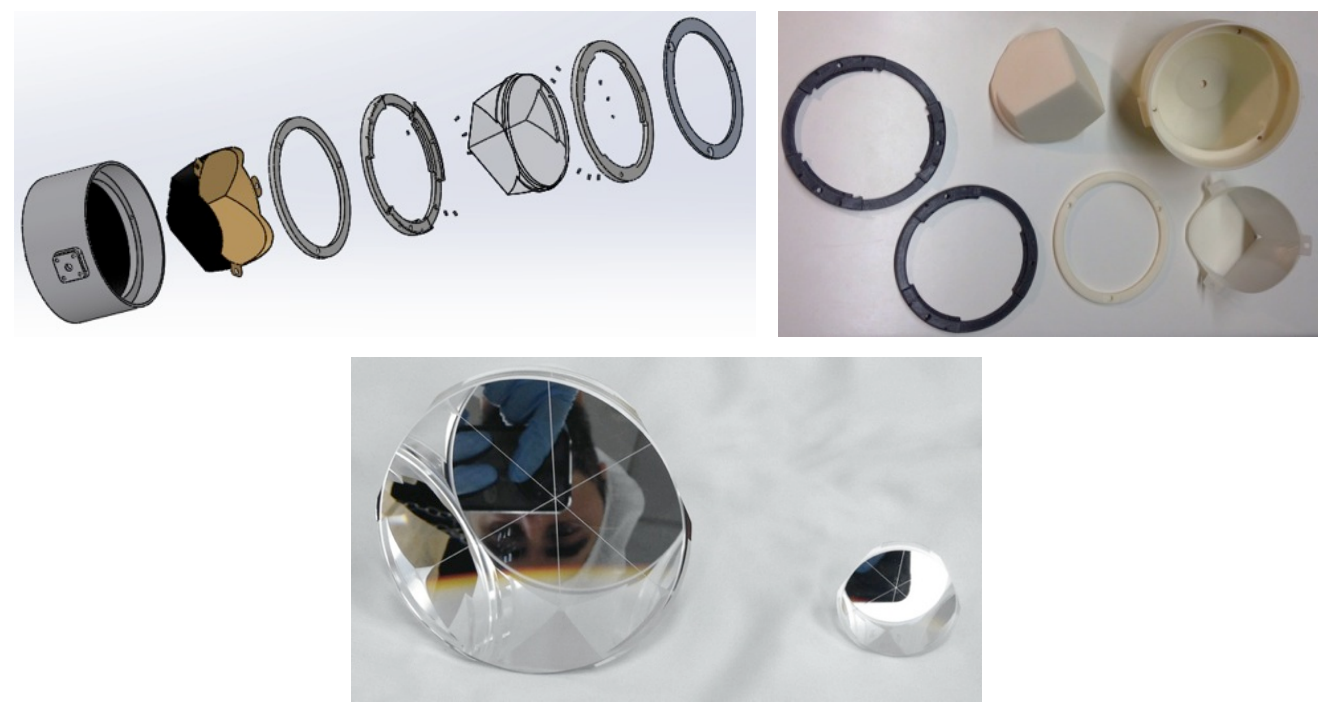

Figure 4. Exploded view of MoonLIGHT (top left). Photos of 3D-printed plastic components of MoonLIGHT (top right). Photo of the MoonLIGHT CCR and of an Apollo CCR (bottom).

Table 1. Precision LLR tests of General Relativity: present and future.

\begin{tabular}{|c|c|c|c|}
\hline \multirow{2}{*}{ Precision test of violation of General Relativity } & \multirow{2}{*}{ Apollo/Lunokhod few cm accuracy [9] } & \multicolumn{2}{|c|}{3 MoonLIGHTs } \\
\hline & & $1 \mathrm{~mm}$ & $0.1 \mathrm{~mm}$ \\
\hline Parameterized Post-Newtonian (PPN) $\beta$ & $|\beta-1|<1.1 \times 10^{-4}$ & $10^{-5}$ & $10^{-6}$ \\
\hline Weak Equivalence Principle (WEP) & $|\Delta a / a|<1.4 \times 10^{-13}$ & $10^{-14}$ & $10^{-15}$ \\
\hline Strong Equivalence Principle (SEP) & $|\eta|<4.4 \times 10^{-4}$ & $3 \times 10^{-5}$ & $3 \times 10^{-6}$ \\
\hline Time Variation of Gravitational Constant (Gdot/G) & $|\dot{G} / G|<9 \times 10^{-13} \mathrm{yr}^{-1}$ & $5 \times 10^{-14}$ & $5 \times 10^{-15}$ \\
\hline Inverse-Square Law (ISL),Yukawa potential & $|\alpha|<3 \times 10^{-11}$ & $10^{-12}$ & $10^{-13}$ \\
\hline Geodetic Precession (GP) & $\left|K_{\mathrm{GP}}\right|<6.4 \times 10^{-3}$ & $6.4 \times 10^{-4}$ & $6.4 \times 10^{-5}$ \\
\hline
\end{tabular}


Development of new gravitational physics models and set experimental constraints using also laser ranging and laser reflectors in the solar system:

- Extension of General Relativity to include Spacetime Torsion [13] [14].

- Non-Minimally Coupled (NMC) gravity, non-minimal gravity coupling between matter and curvature, also called " $f_{1}(R)+f_{2}(R)$ " theories [15] [16].

\section{LRA for Solar System Exploration and Geodesy}

We propose INRRI both for landers and rovers on Moon and Mars. The following description is for Mars.

- INRRI (shown in Figure 5) is a laser retroreflector micropayload of about 50 gr weight and about $55 \mathrm{~mm} \mathrm{x}$ $20 \mathrm{~mm}$ size. It will be laser tracked by Mars orbiters capable of laser ranging and/or laser altimetry, like for example LOLA (Lunar Orbiter Laser Altimeter) on LRO (Lunar Reconnaissance Orbiter) and/or laser communication, like for example LLCD (Lunar Laser Comm demo) on LADEE (Lunar Atmosphere and Dust Environment Explorer), or OPALS (Optical PAyload for Lasercomm Science) on the ISS. INRRI is developed for US Mars landers and rovers and on European landers and rovers with Italian interest and/or involvement. INRRI has been sized to give the right signal for LRO

o In addition: laser flashes sent by orbiters and retroreflected back by INRRI can also be observed by optical cameras on the same orbiters. If INRRI would be now on the Moon at the poles LOLA receiver would see it (ordinary laser ranging/altimetry time-of-flight), and so probably also LROC (camera of LRO).

- Deploying multiple INRRIs on landers and rover will lead over time to the establishment of the retroreflector components of a Mars Geophysics Network. The location of the Prime Meridian of Mars can be defined very effectively by an INRRI-equipped lander, or rover at EoL, laser-located by Mars orbiters (perhaps a future, Mars-adapted version of LOLA, whose more accurate mapping will replace laser altimetry maps of MOLA, Mars Orbiter Laser Altimeter). Currently the Prime Meridian is defined by the Airy-0 crater with an accuracy of the order of $50 \mathrm{~m}$; with INRRI it could be defined with the accuracy better by orders of magnitude. Deployment of three or more LRA of the INRRIs on Mars will allow for triangulations by orbiters.

- INRRI for Mars Rovers is a new enabling technology for planetary exploration because: it will provide accurate Rover geo-referencing during its exploration activity, recording its positions where significant geological measurements have been made by Rover instruments as reference for future exploratory missions. Example of the latter: future sample return mission targeting an INRRI-georeferenced position explored/ surveyed by the Rover of particularly high interest to NASA/ESA/ASI long-term goals for Mars exploration (outstanding astrobiologically relevant sites, potential biosignature locations).

- The rover/lander with INRRI will also be a passive, wavelength-independent, long-lived reference point enabling the performance of full-column measurement of trace species in the Mars atmosphere by future space-borne lidars. This will be complementary to highly localized measurements made by gas sampling techniques on the rover or by laser back-scattering lidar techniques on future orbiters and/or from the surface.
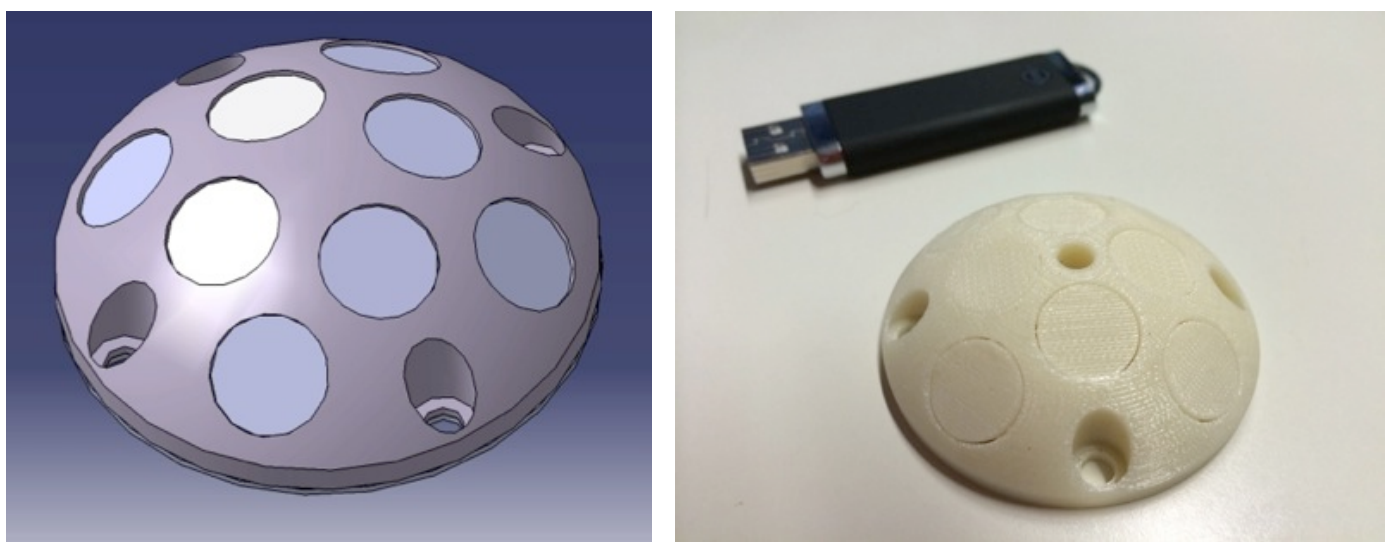

Figure 5. Drawing and photo of INRRI, Instruments for landing/Roving laser Retroreflector Investigations. 
- INRRI for Mars Rovers is a new, wavelength-independent, enabling technology to test, validate, and locally diagnose, on Mars, certain aspects related to transmitter and receiver sub-subsystems for future laser-communication from Mars orbiters to Earth, an activity that is a long-term interest for future Mars missions. This will be also applied to future laser-comm between Mars orbiters and Mars surface (future Rovers and distributed installations).

- INRRI for Mars will also support future technology experiments of quantum laser communication exploiting the polarization states of laser photons, carried out among future Mars orbiters and the Mars Rovers.

- In summary, laser measurements by future orbiters with INRRI are:

o Time-of-flight laser altimetry

o Time-of-flight ranging from space to (Mars) ground: “inverse SLR network”, which will be an unprecedented activity. In fact, while to SLR/LLR has been done only by ILRS ground stations, no orbiting laser payload has ever ranged to a CCR outside Earth. The LOLA altimeter did not range to Apollo arrays because its receivers would have been damaged by too large a laser return. INRRIs on Moon and Mars surfaces need to be specially designed, with appropriately small optical cross section (and size), in order to be laser ranged by orbiters.

o Rover geo-referencing during its exploration activity by laser altimetry and laser ranging

o Lidar atmospheric trace species detection

o Laser-communication test and diagnostics.

INRRI and/or appropriately adapted versions of INRRI are suited for deployment on the icy/rocky moons of Jupiter/Saturn: these payloads will be called ECCE-INRRI (Europa/Enceladus Cube Corners for Exploration and Exolife-Instruments for landing/Roving laser Retroreflector Investigations).

The conceptual Figure 6 summarizes the laser tracking of INRRI deployed on the Moon, Mars, Jupiter/Saturn moons or asteroid:

- Selenolocate Lander/Rover with laser retroreflector by:

o Laser Altimetry at nadir (LRO-like) to rovers/landers at poles of moon(s)

o Laser Ranging/Laser-Comm to reflectors anywhere, like LLCD, OPALS and iROC (integrated Radio and Optical Communications, under development by NASA-GRC).

- Deploy INRRI networks; also on far side of Earth's Moon.

We are also considering INRRI, or its variations, for applications to Near Earth Asteroids (NEAs) and/or Near Earth Objects (NEOs) that are of interest to ESA, NASA and the European HORIZON2020 program.

\section{GNSS}

Our work on GNSS with NASA, ASI, ESA and ISRO is consolidated and reported in [1] [2] [17].

Within the R\&D ASI-INFN project dedicated to GNSS and to Galileo in particular, called ETRUSCO-2 (Extra Terrestrial laser Ranging to Unified GNSS Satellite Constellations-Phase 2, [2] [17]) we designed, built and characterized a standard GRA using the consolidated fused silica retroreflector technology space-qualified first with Apollo LRAs and later with LAGEOS. Some of the criteria used in the GRA design are reported schematically in Figure 7, right. Some of these are the ones endorsed by ILRS and reported in [1]. The GRA is shown inside the SCF-G in Figure 7. The GRA has been characterized with the SCF-G, according to the procedures

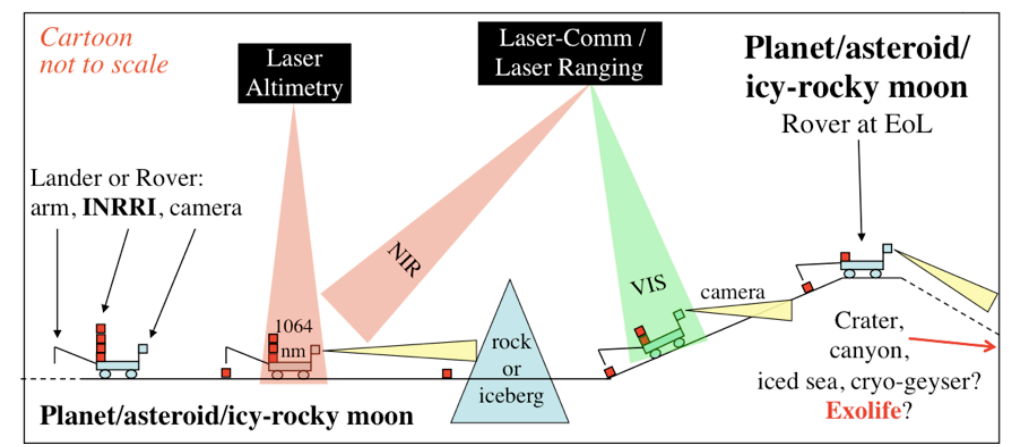

Figure 6. Graphical sketch describing conceptual deployment of INRRI in the solar system. 


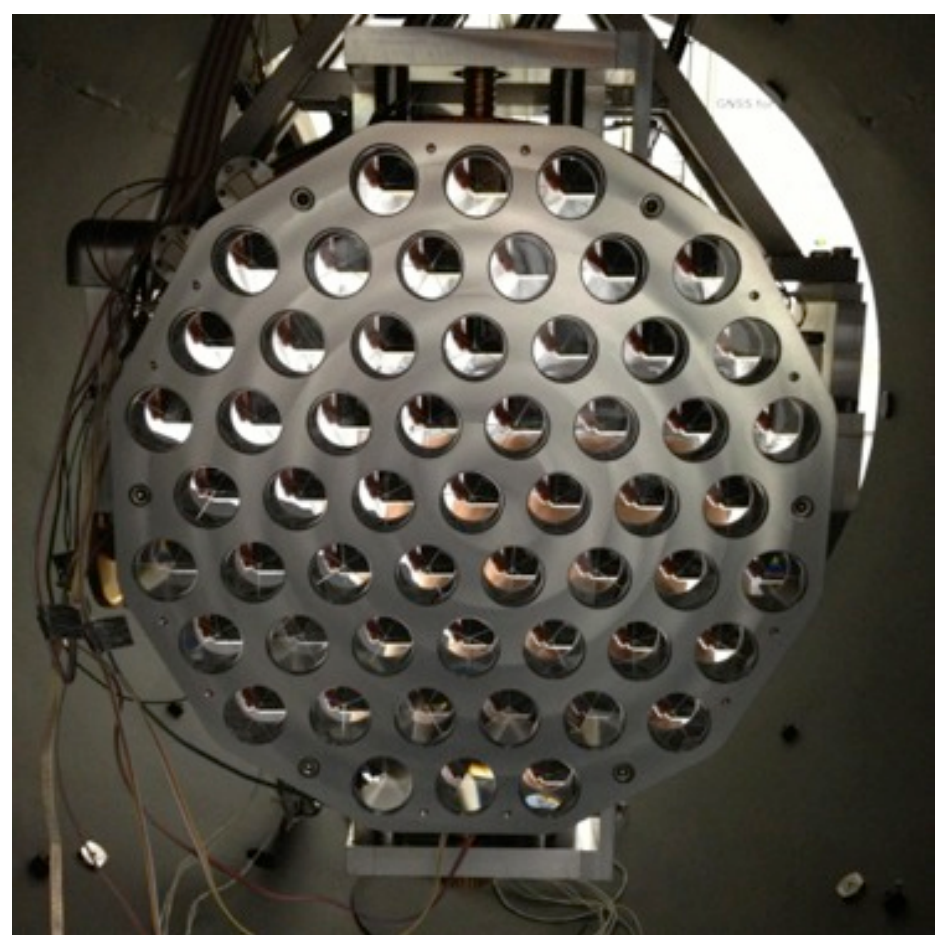

Figure 7. Left: GNSS Retroreflector Array, GRA (55 uncoated CCR) in the SCF-G.

foreseen by the SCF-Test/Revision-ETRUSCO-2, which include investigation of the GRA optical performance and thermal behavior along the GCO, under exposure to the solar simulator illumination (Figure 8, and [2] for more details). The GRA optical performance has been assessed in terms of FFDPs (Figure 9) and WFIs of its CCRs. The thermal behavior has been determined with IR thermometry and contact probes.

A single Galileo IOV CCR provided by ESA/Galileo has been GCO-SCF-Tested in 2010; his optical performance, reported in Figure 10, shows thermal degradations (of about 35\%, averaged over the whole GCO), while the GRA (Figure 9) has no average degradation. However, more spare Galileo IOV flight CCRs have been SCF-Tested in Frascati (Figure 11(a)), under a dedicated ESA-INFN contract to be completed in January 2014. The $5^{\text {th }}$ Galileo IOV flight-quality LRA (Figure 11(b)) is currently in Frascati to be characterized in the framework of a joint ASI-INFN project funded by the Italian Ministry of Instruction, University and Research.

\section{Earth Observation}

We are developing a midsize LRA suited for LEO and EO constellations like ESA Sentinels and, in general, for the space segment of Copernicus (European Flagship space program, also part of HORIZON2020). One model is shown in Figure 12, which is one co-developed and co-studied by INFN and the Italian Ministry of Defense and the Italian Ministry of Foreign Affairs and International Cooperation (high-relevance Italy-USA bilateral project AUGUSTUS-2014, Absolute crust, Glacier and iceberg Georeferencing with Unified Sar, optical, gnss laser observations by Italy and USa-2014).

CORA and/or its appropriately adapted versions are suited for deployment as Phobos AND Deimos laser Retroreflector Arrays (PANDORAs).

\section{National and International Framework}

We work in strong synergism with the ground stations of ILRS, in particular with the Matera Laser Ranging Observatory in Italy, of the ASI-CGS. We collaborate with ASI, NASA, ESA and ISRO. We are submitting proposals to Roscosmos and other space agencies. INFN is an Affiliate Partner of the NASA-SSERVI (Solar System Exploration Research Virtual Institute) for research themes reported in this paper, with S. Dell'Agnello as Principal Investigator (see http://sservi.nasa.gov/internationals/). 


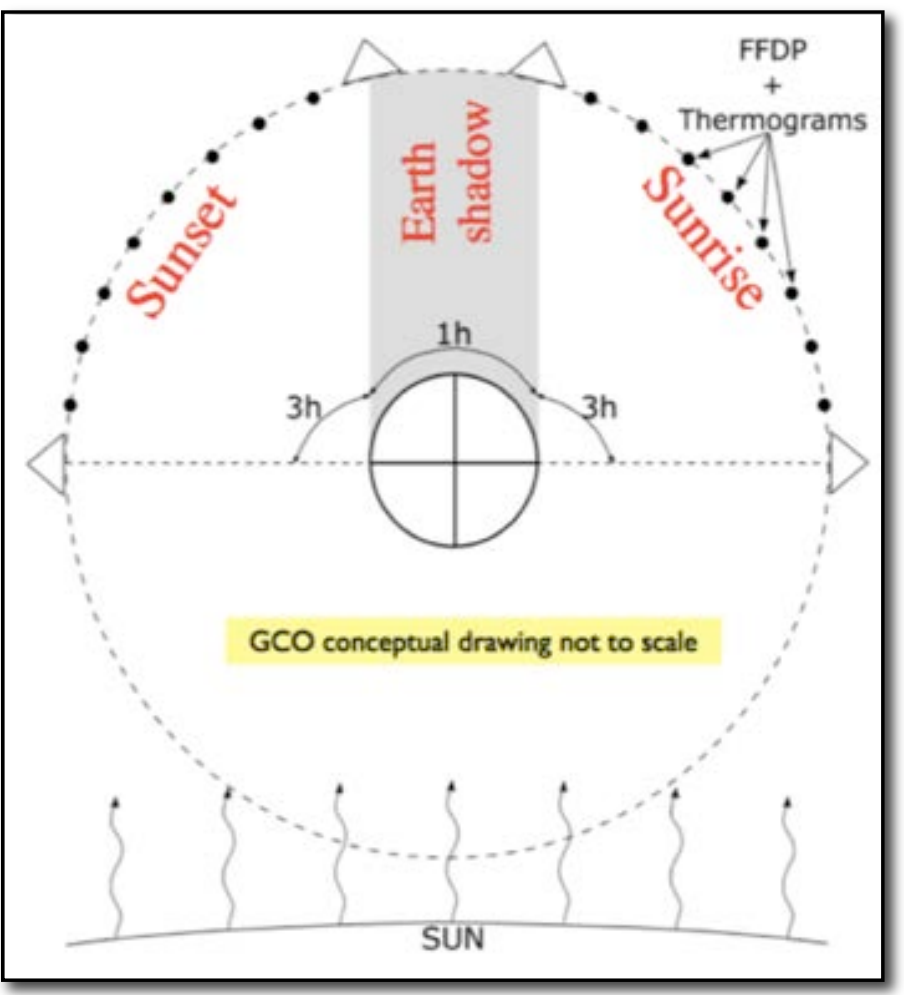

Figure 8. Right: graphical sketch of the GCO test.

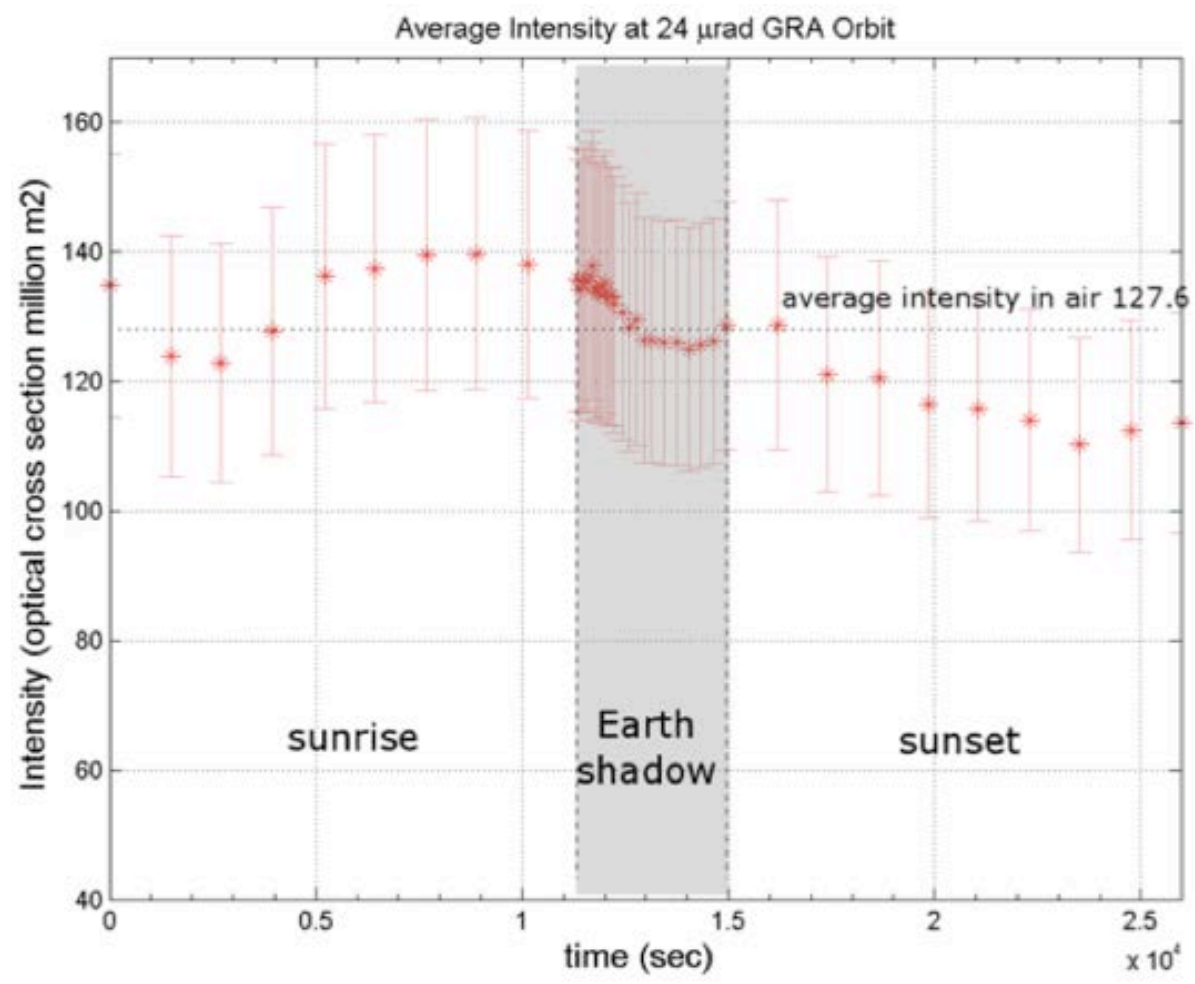

Figure 9. FFDP intensity of GRA during GCO test compared to the nominal performance in air and isothermal conditions: the GRA developed by INFN shows, on average, no performance degradation under SCF-Test. 


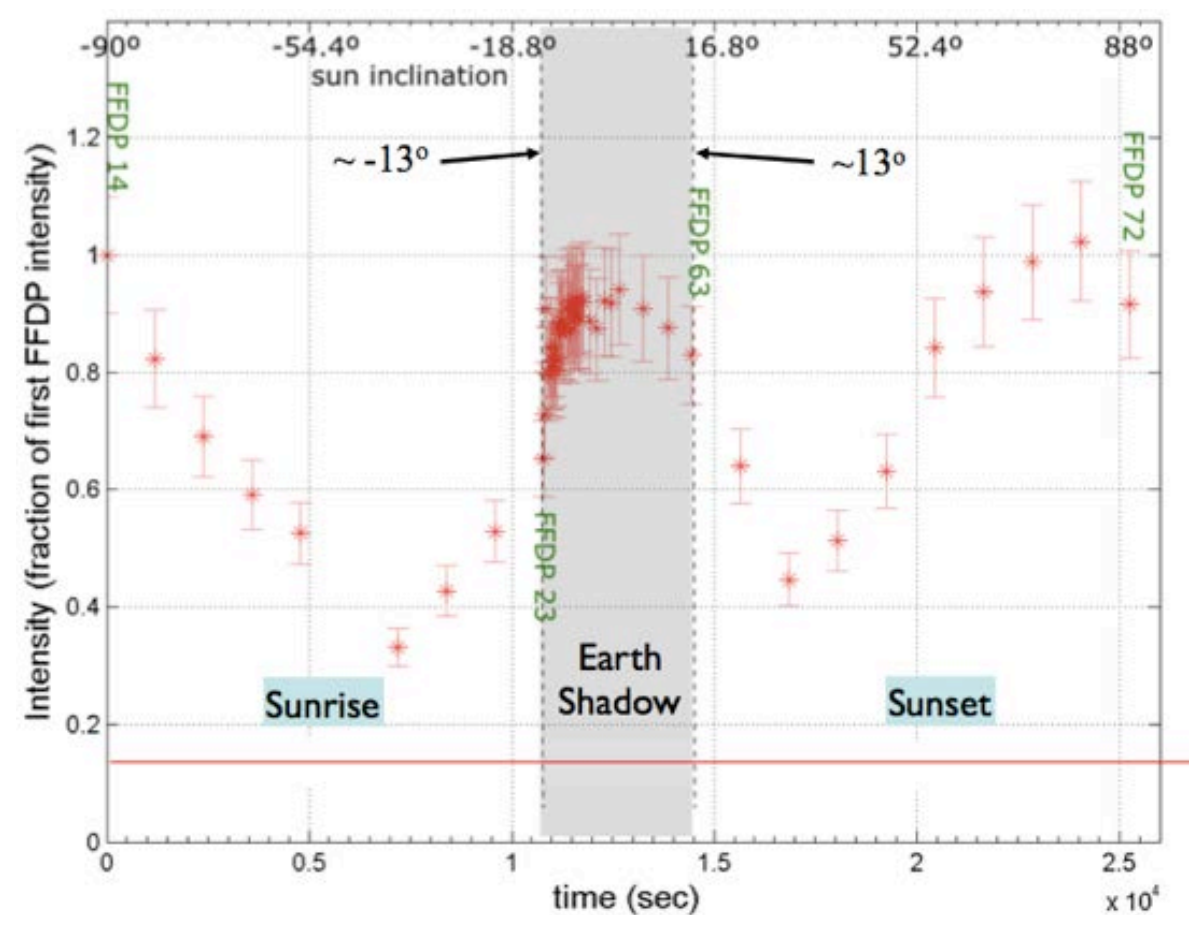

Figure 10. FFDP intensity of a single Galileo IOVCCR during the GCO test of 2010 [2], showing on average a performance degradation by $\sim 35 \%$. The red line shows the GPS/ GLONASS/GIOVE degradation by 85\% [1].
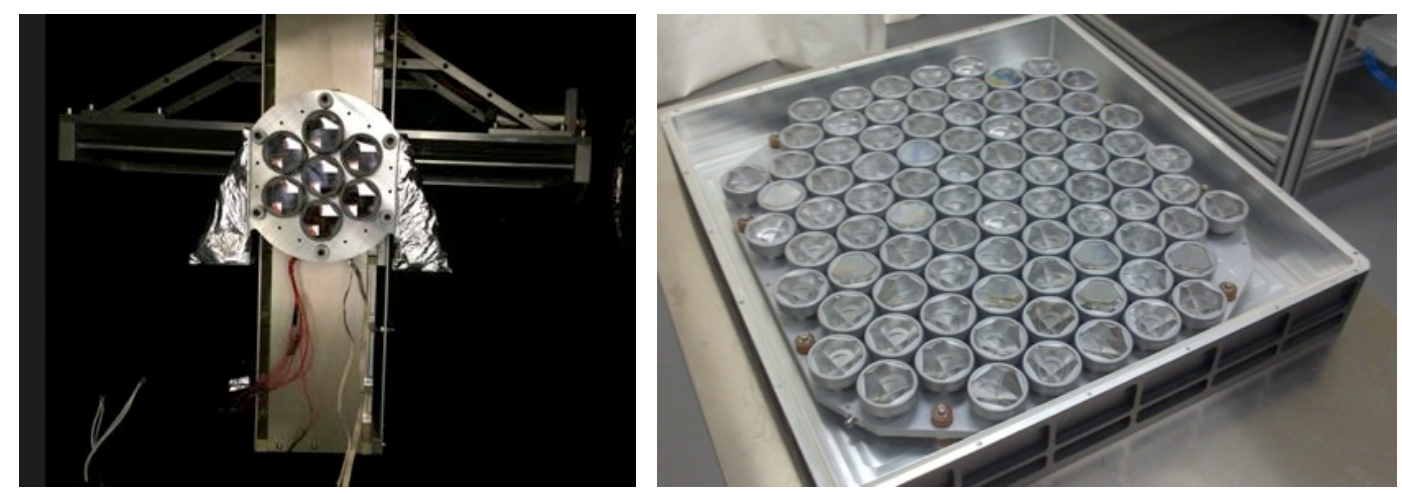

Figure 11. GRA (55 uncoated CCR) on the roto-translation, positioning and thermal control system in SCF-G.

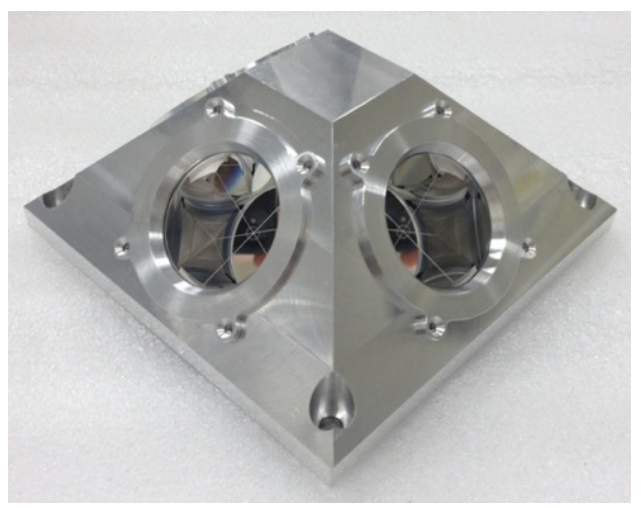

Figure 12. Model of CORA, COpernicus laser Retroreflector Array. 
Within INFN, this work is carried out in the INFN National Scientific Committees n. 2 (CSN2, including space science) and n. 5 (CSN5, including space technologies), and in collaboration of ASI. Some activities are carried out with co-funding (mentioned in the previous sections) by the Italian: Ministry of Instruction University and Research (MIUR), of the Ministry of Defense (MD) and of the Ministry of Foreign Affairs and International Cooperation (MAECI).

Work done for space ASI, ESA and Italian Ministries is reported in [17].

\section{References}

[1] Dell'Agnello, S., et al. (2011) Creation of the New Industry-Standard Space Test of Laser Retroreflectors for the GNSS and LAGEOS. J. Adv. Space Res., 47, 822-842. http://ilrs.gsfc.nasa.gov/about/reports/other_publications.html http://dx.doi.org/10.1016/j.asr.2010.10.022

[2] Dell'Agnello, S., et al. (2011) ETRUSCO-2, an ASI-INFN Project for the Development and SCF-Test of GNSS Laser Retroreflector Arrays. ESA Proceedings of $3^{\text {rd }}$ International Colloquium-Scientific and Fundamental Aspects of the Galileo Programme, Copenhagen. http://ilrs.gsfc.nasa.gov/missions/satellite_missions/current_missions/ga01_reflector.html

[3] Boni, A., et al. (2011) World-first SCF-Test of the NASA-GSFC LAGEOS Sector and Hollow Retroreflector. Proc. 17th International Workshop on Laser Ranging, Bad Kötzting, Germany.

[4] Bosco, A., et al. (2007) Probing Gravity in NEO’s with High-Accuracy Laser-Ranged Test Masses. Int. Jou. Mod. Phys. D, 16, 2271-2285. http://dx.doi.org/10.1142/S0218271807011322

[5] Bender, P.L., et al. (1973) The Lunar Laser Ranging Experiment. Science, 182, 229-238. http://dx.doi.org/10.1126/science.182.4109.229

[6] Shapiro, I.I., Reasenberg, R.D., Chandler, J.F. and Babcock, R.W. (1988) Measurement of the de Sitter Precession of the Moon: A Relativistic Three-Body Effect. Phys. Rev. Lett., 61, 2643. http://dx.doi.org/10.1103/PhysRevLett.61.2643

[7] Williams, J.G., Turyshev, S.G. and Boggs, D.H. (2004) Phys. Rev. Lett., 93, 261101. http://dx.doi.org/10.1103/PhysRevLett.93.261101

[8] Currie, D., Dell’Agnello, S. and Delle Monache, G. (2011) A Lunar Laser Ranging Retroreflector Array for the $21^{\text {st }}$ Century. Acta Astronaut, 68, 667-680. http://dx.doi.org/10.1016/j.actaastro.2010.09.001

[9] Martini, M., et al. (2012) MoonLIGHT: A USA-Italy Lunar Laser Ranging Retroreflector Array for the $21^{\text {st }}$ Century. Planetary and Space Science, 74, 276-282. http://dx.doi.org/10.1016/j.pss.2012.09.006

[10] Dell'Agnello, S., et al. (2012) Probing General Relativity and New Physics with Lunar Laser Ranging. Nuclear Instruments and Methods in Physics Research A, 692, 275-279. http://dx.doi.org/10.1016/j.nima.2012.01.002

[11] Currie, D., Dell’Agnello, S., Delle Monache, G., Behr, B. and Williams, J.G. (2013) A Lunar Laser Ranging Retroreflector Array for the $21^{\text {st }}$ Century. Nuclear Physics B (Proc. Suppl.), 243-244, 218-228. http://dx.doi.org/10.1016/j.nuclphysbps.2013.09.007

[12] Dell'Agnello, S., et al. (2011) Fundamental Physics and Absolute Positioning Metrology with the MAGIA Lunar Orbiter. Phase A Study for ASI's Call for Small Missions. Exp. Astron., 32, 19-35. http://dx.doi.org/10.1007/s10686-010-9195-0

[13] March, R., Bellettini, G., Tauraso, R. and Dell'Agnello, S. (2011) Constraining Spacetime Torsion with the Moon and Mercury. Phys. Rev. D, 83, 104008. http://dx.doi.org/10.1103/PhysRevD.83.104008

[14] March, R., Bellettini, G., Tauraso, R. and Dell’Agnello, S. (2011) Constraining Spacetime Torsion with LAGEOS. Gen. Relativ. Gravit., 43, 3099-3126. http://dx.doi.org/10.1007/s10714-011-1226-2

[15] Bertolami, O., March, R. and Paramos, J. (2013) Solar System Constraints to Non-Minimally Coupled Gravity. Phys. Rev. D, 88, 064019. http://dx.doi.org/10.1103/PhysRevD.88.064019

[16] Castel-Branco, N., Paramos, J. and March, R. (2014) Perturbation of the Metric around a Spherical Body from a Nonminimal Coupling between Matter and Curvature. Phys. Rev. B, 735, 25-32.

[17] Dell'Agnello, S., et al. (2013) A Unique Infrastructure to Develop and SCF-Test Laser Retroreflector Arrays for GNSS, EGNOS-V2 and Inter-Gnss-Satellite Laser Links. ESA Proceedings of $4^{\text {th }}$ International Colloquium-Scientific and Fundamental Aspects of the Galileo Programme, Prague. 